\title{
A SIMPLE PROOF OF THE GOLDBERG-STRAUS THEOREM ON NUMERICAL RADII
}

\author{
by BIT-SHUN TAM
}

(Received 6 March, 1985)

1. Introduction. Let $M_{n}(\mathbb{C})$ be the algebra of $n \times n$ complex matrices, and let $U_{n}$ be its unitary group. Given $A, B \in M_{n}(\mathbb{C})$, the $A$-numerical radius of $B$ is the nonnegative quantity

$$
r_{A}(B)=\max \left\{\mid \operatorname{tr}\left(A U^{*} B U \mid: U \in \mathcal{U}_{n}\right\} .\right.
$$

In particular, for $A=\operatorname{diag}(1,0, \ldots, 0)$ it reduces to the classical numerical radius $r(B)=\max \left\{\left|x^{*} B x\right|: x^{*} x=1\right\}$. In [1] Goldberg and Straus proved that $r_{A}$ is a generalized matrix norm (i.e. a positive definite seminorm) on $M_{n}(\mathbb{C})$ if and only if $A$ is nonscalar and $\operatorname{tr} A \neq 0$. This result agrees with the well-known fact that the classical numerical radius $r$ is a generalized matrix norm. The nontrivial part of the proof is to show that if $A$ is nonscalar and $\operatorname{tr} A \neq 0$ then $r_{A}$ is positive definite; that is, for any $B \in M_{n}(\mathbb{C})$, $\operatorname{tr}\left(A U^{*} B U\right)=0$ for all $U \in \mathcal{U}_{n}$ implies $B=0$. The proof given in [1] is computational and involves the use of differentiation on matrices. Later Marcus and Sandy [2] gave three elementary proofs of the result. Their proofs are still computational in nature and two of them need knowledge of multilinear algebra.

In this note we will give an easier and more conceptual proof of the result. The ideas of our proof stem from a simple observation. In terms of the usual inner product on $M_{n}(\mathbb{C})$ defined by $\langle A, B\rangle=\operatorname{tr}\left(A B^{*}\right)$, the condition " $\operatorname{tr}\left(A U^{*} B U\right)=0$ for all $U \in U_{n}$ " is equivalent to saying that $B^{*}$ belongs to the orthogonal complement of the linear span of $\left\{U A U^{*}: U \in U_{n}\right\}$. Thus, the nontrivial part of the above result of Goldberg and Straus says:

If $A$ is nonscalar and $\operatorname{tr} A \neq 0$, then $\operatorname{span}\left\{U A U^{*}: U \in \mathcal{U}_{n}\right\}=M_{n}(\mathbb{C})$. In the course of our proof we reduce the problem to proving the geometric fact stated in Theorem 1 . It turns out that Theorem 1 and the result of Goldberg and Straus are "equivalent".

In [2] Marcus and Sandy also posed a conjecture which extends the above result of Goldberg and Straus. In [4] the conjecture is shown to be incorrect. We will also give some related results in [3].

2. Proofs of results. For any $A \in M_{n}(\mathbb{C})$, by a unitary (permutational) transform of $A$ we mean a matrix of the form $U^{*} A U$, where $U \in \mathcal{U}_{n}$ ( $U$ is a permutation matrix).

Lemma. Let $C, D \in M_{n}(\mathbb{C})$ such that $\operatorname{tr} C=\operatorname{tr} D=0$ and $C \neq 0$. If there exists a constant $\alpha$ such that, for all $U \in \mathcal{U}_{n}\left\langle C, U^{*} D U\right\rangle=\alpha$, then $D=0$.

Proof. By the fact $\operatorname{tr}(A B)=\operatorname{tr}(B A)$, it is easily shown that in the condition

$$
\left\langle C, U^{*} D U\right\rangle=\alpha \text { for all } U \in U_{n}
$$

we may replace $C$ and $D$ by their unitary transforms. Also note that there is a unitary

Glasgow Math. J. 28 (1986) 139-141. 
transform of $C$ with some main diagonal entries being nonzero; for instance, if $c_{i i}=0$ for $1 \leq i \leq n$ and $c_{12}$ or $c_{21} \neq 0$, then there exists some real number $\theta$ such that the $(1,1)$ and $(2,2)$ entries of the following unitary transform of $C$ are nonzero:

$$
\left[\left(\begin{array}{cc}
1 / \sqrt{2} & -e^{-i \theta} / \sqrt{2} \\
1 / \sqrt{2} & e^{-i \theta} / \sqrt{2}
\end{array}\right) \oplus I_{n-2}\right] C\left[\left(\begin{array}{cc}
1 / \sqrt{2} & 1 / \sqrt{2} \\
-e^{i \theta} / \sqrt{2} & e^{i \theta} / \sqrt{2}
\end{array}\right) \oplus I_{n-2}\right] .
$$

Let $\tilde{C}=\left(1 / 2^{n}\right) \sum U C U^{*}$, where the summation is taken over all $n \times n$ diagonal matrices $U$ with 1 or -1 along its main diagonal. (There are altogether $2^{n}$ such matrices.) It is not difficult to show that $\tilde{C}$ is a diagonal matrix with the same main diagonal as $C$. Further, (1) still holds if $C$ is replaced by $\tilde{C}$.

So, from the above observations we may assume that $C$ is a nonzero diagonal matrix with zero trace. Replacing $C$ by a permutational transform, if necessary, we may assume that $c_{11}, c_{22}$ are nonzero, unequal entries of $C$. Now suppose that $D$ is nonzero. Again replacing $D$ by a suitable unitary transform, we may assume that $d_{11}, d_{22}$ are nonzero and unequal. Now by (1),

$$
\alpha=\operatorname{tr}\left(C D^{*}\right)=c_{11} \bar{d}_{11}+c_{22} \bar{d}_{22}+\sum_{i=3}^{n} c_{i i} \bar{d}_{i i} .
$$

As the matrix $\operatorname{diag}\left(c_{22}, c_{11}, c_{33}, \ldots, c_{n n}\right)$ is permutationally similar to $C$, by (1) again we also have,

$$
\alpha=c_{11} \bar{d}_{22}+c_{22} \bar{d}_{11}+\sum_{i=3}^{n} c_{i i} \bar{d}_{i i}
$$

Hence, $\quad c_{11} \bar{d}_{11}+c_{22} \bar{d}_{22}=c_{11} \bar{d}_{22}+c_{22} \bar{d}_{11} ; \quad$ or $\quad\left(c_{11}-c_{22}\right)\left(\bar{d}_{11}-\bar{d}_{22}\right)=0$, which is a contradiction.

Since hyperplanes in the subspace $\left\{D \in M_{n}(\mathbb{C}): \operatorname{tr} D=0\right\}$ are of the form $\{D \in$ $M_{n}(\mathbb{C}): \operatorname{tr} D=0$ and $\left.\langle D, C\rangle=\alpha\right\}$ for some constant $\alpha$ and some matrix $C$ with zero trace, the above lemma can be restated in the following geometric language.

TheOREM 1. Suppose that $A \in M_{n}(\mathbb{C})$ is a nonzero matrix with zero trace. Then the complex affine hull of the set $\left\{U A U^{*}: U \in \mathscr{U}_{n}\right\}$ is equal to the subspace of matrices with zero trace.

(The complex affine hull of a nonempty set $S$ consists of all vectors of the form $\alpha_{1} x_{1}+\ldots+\alpha_{p} x_{p}$, where $x_{i}$ are vectors in $S, \alpha_{i}$ are (complex) scalars such that $\sum_{i=1}^{p} \alpha_{i}=1$ and $p$ is some positive integer.)

Theorem 2 (Goldberg-Straus). If $A \in M_{n}(\mathbb{C})$ is nonscalar and $\operatorname{tr} A \neq 0$, then $\operatorname{span}\left\{U A U^{*}: U \in \mathscr{U}_{n}\right\}=M_{n}(\mathbb{C})$.

Proof. Express $A$ in the form $C+\alpha I_{n}$ where $C=A-(1 / n)(\operatorname{tr} A) I_{n}$ and $\alpha=$ $=(1 / n) \operatorname{tr} A$. Then clearly $C$ is a matrix with zero trace and is nonzero as $A$ is nonscalar. By Theorem 1, for any $D \in M_{n}(\mathbb{C})$ with zero trace, there exist $U_{i} \in \mathcal{U}_{n}$ and scalars 
$\alpha_{i}, 1 \leq i \leq p$, with $\sum_{i=1}^{p} \alpha_{i}=1$ such that $D=\alpha_{1} U_{1} C U_{1}^{*}+\ldots+\alpha_{p} U_{p} C U_{p}^{*}$; then $D^{-}+\alpha I_{n}=$ $\alpha_{1} U_{1} A U_{1}^{*}+\ldots+\alpha_{p} U_{p} A U_{p}^{*}$. This shows that $\operatorname{span}\left\{U A U^{*}: U \in U_{n}\right\}$ contains the hyperplane $\left\{D \in M_{n}(\mathbb{C}): \operatorname{tr} D=0\right\}+\alpha I_{n}$. As $\alpha \neq 0$, this hyperplane is not a hypersubspace and hence spans $M_{n}(\mathbb{C})$. It follows that $\operatorname{span}\left\{U A U^{*}: U \in U_{n}\right\}=M_{n}(\mathbb{C})$.

We have deduced Theorem 2 from Theorem 1. For completeness we show below that Theorem 1 also follows from Theorem 2.

If Theorem 1 is incorrect, then so is our lemma. Then there exist nonzero matrices $C, D \in M_{n}(\mathbb{C})$ both with zero trace such that, for all $U \in U_{n},\left\langle C, U^{*} D U\right\rangle=\alpha$ for some constant $\alpha$. Denote the matrix $D+I_{n}$ by $A$. Then $A$ is nonscalar and tr $A \neq 0$. Further, $\operatorname{span}\left\{U A U^{*}: U \in \mathscr{U}_{n}\right\}$ is a proper subspace of $M_{n}(\mathbb{C})$, because for any $U \in \mathscr{U}_{n}$,

$$
\left\langle C-\frac{\alpha}{n} I_{n}, U^{*}\left(D+I_{n}\right) U\right\rangle=\left\langle C, U^{*} D U\right\rangle-\left\langle\frac{\alpha}{n} I_{n}, I_{n}\right\rangle=0,
$$

whereas $C-(\alpha / n) I_{n} \neq 0$. This shows that Theorem 2 does not hold, which is a contradiction.

\section{REFERENCES}

1. M. Goldberg and E. G. Straus, Norm properties of $C$-numerical radii, Linear Algebra and Appl. 24 (1979), 113-131.

2. M. Marcus and M. Sandy, Three elementary proofs of the Goldberg-Straus theorem on numerical radii, Linear and Multilinear Algebra 11 (1982), 243-252.

3. B. S. Tam, The action of unitary transforms of a matrix on linear subspaces, submitted for publication.

4. T. Y. Tam, On the generalized radial matrices and a conjecture of Marcus and Sandy, Linear and Multilinear Algebra, to appear.

Department OF Mathematics

TAMKANG UNIVERSITY

Taipei, Taiwan 251

RePublic of China 